\title{
Serial Block Face-Scanning Electron Microscopy of the Developing Rat Brain Exposed to Ketamine Reveals Changes in Mitochondrial Ultrastructure.
}

Trisha Eustaquio ${ }^{1}$, Cheng Wang ${ }^{2}$, Christopher K. Dugard ${ }^{1}$, Nysia I. George ${ }^{2}$, Fang Liu ${ }^{2}$, William

Slikker Jr. ${ }^{2}$, Merle G. Paule ${ }^{2}$, Paul Howard ${ }^{2}$ and $\underline{\text { Angel Paredes }}{ }^{2}$

1. U.S. Food and Drug Administration, Center for Devices and Radiological Health, Silver Spring, Maryland, USA

2. U.S. Food and Drug Administration, National Center for Toxicological Research, Jefferson, Arkansas, USA

In the brains of experimental animals there is accumulating evidence that prolonged exposure to ketamine, a N-methyl-D-aspartate (NMDA) receptor antagonist, causes widespread apoptotic cell death [1]. Understanding the long term impact of ketamine exposure on human health and in particular on child development is critical as ketamine is commonly used as a general anesthetic on young children undergoing certain medical procedures such as surgery. It is generally accepted that mitochondria play a pivotal role in apoptosis, a vital and necessary biological pathway used by many organisms for programed cell death. This role may be inadvertently triggered by ketamine causing neurotoxicity by inducing inappropriate apoptosis in affected neurons. Ketamine-induced alterations in mitochondria may be associated with intracellular signals that cause effected neurons to initiate apoptosis.

In this study, we examined the brain damage accompanying ketamine exposure (20 mg/kg bw, subcutaneously every $2 \mathrm{hr}$ for $12 \mathrm{hr}$; $12 \mathrm{hr}$ recovery) in the frontal cortex of Sprague-Dawley rats (postnatal day 7). Initially, conventional transmission electron microscopy (TEM) was used to examine the overall mitochondrial structure in 2D in order to follow and grade the changes that occur within mitochondria after treatment versus control. Using a grading scale based on cristae conformation, there is a clear difference $(\mathrm{P}<0.05)$ in the mitochondria of ketamine-treated versus control animals.

In order to relate images obtained by conventional TEM to the overall structural effect this drug has on brain tissue, serial block face scanning electron microscopy (SBF-SEM) was used to examine the 3D structure of 10-20 $\mu^{3}$ volumes of frontal cortex [2]. Segmentation of individual mitochondria from both untreated control and ketamine treated tissue reconstructed from SBF-SEM data allowed us to extract and compare individual mitochondria from the 3D volumes. We found that mitochondria in healthy control tissue were mostly elongated with many forming networks with neighboring mitochondria (Figure 1, left), while the mitochondria in tissue from ketamine-treated rats were spherical and discrete, present in tightly packed groups, lacking interconnectivity (Figure 1, right). Both TEM and SBF-SEM support the hypothesis that ketamine damages mitochondria and this effect is associated with the neurotoxicity observed following ketamine treatment.

The information in these materials is not a formal dissemination of information by the FDA and does not represent agency position or policy.

[1] J Liu, Q Liu, J Li, C Baek, K Han, U Athiraman, and S Soriano, Anesthesiology 117(1) (2012), p.64. [2] W Denk, and H Horstmann, PLoS biology (2004) 


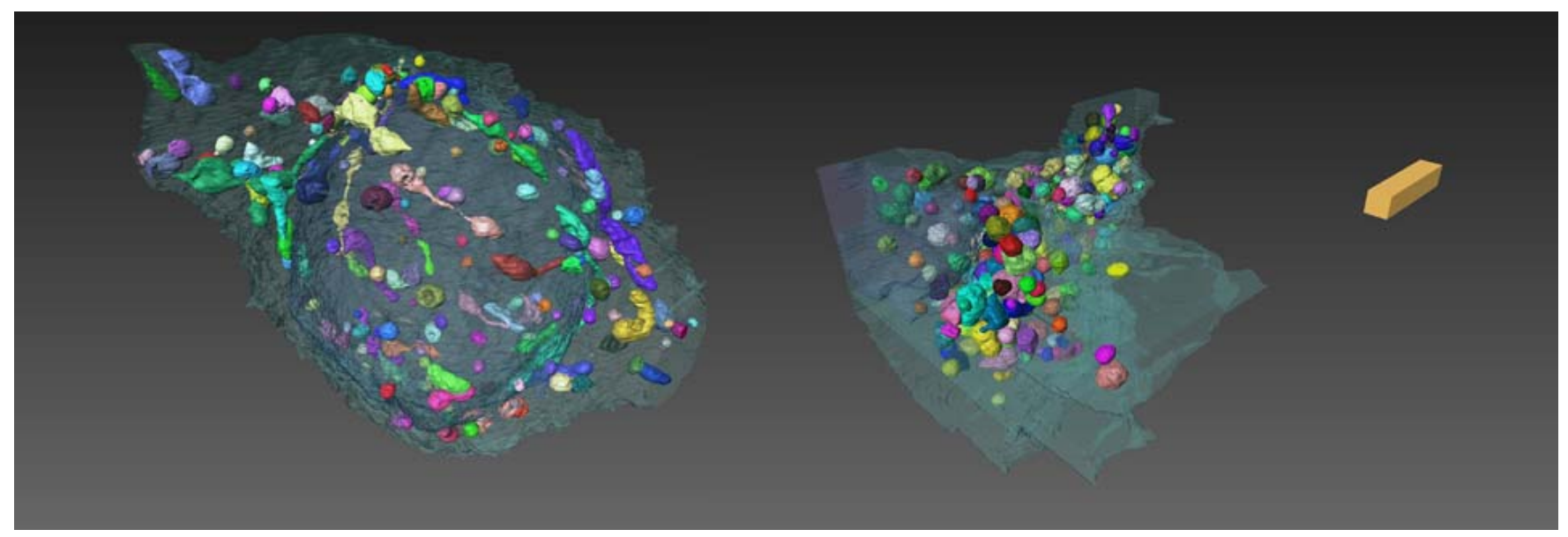

Figure 1. On the left, segmented volume of a neuron extracted from the frontal cortex of an untreated rat brain. On the right, segmented volume of a neuron extracted from the frontal cortex of a ketamine treated (20 mg/kg bw, subcutaneously every 2 hr for 12 hr; 12 hr recovery) rat brain. The mitochondria were segmented in arbitrary colors in order to enhance spatial contrast. The scale box represents 5:1:1 $\mu \mathrm{m}(x: y: z)$ shown in yellow in the top right corner. 